\title{
Literature Alert
}

\author{
Norman K. Hollenberg
}

Published online: 19 January 2010

(C) Springer Science+Business Media, LLC 2010

\begin{abstract}
The beneficial effects of flavonoid consumption on cardiovascular risk are supported by mechanistic and epidemiologic evidence. We aimed to systematically review the effectiveness of different flavonoid subclasses and flavonoidrich food sources on cardiovascular disease (CVD) and risk factors-ie, lipoproteins, blood pressure, and flow-mediated dilatation (FMD). Methods included a structured search strategy on MEDLINE, EMBASE, and Cochrane databases; formal inclusion or exclusion, data extraction, and validity assessment; and meta-analysis. One hundred thirty-three trials were included. No randomized controlled trial studied effects on CVD morbidity or mortality. Significant heterogeneity confirmed differential effects between flavonoid subclasses and foods. Chocolate increased FMD after acute (3.99\%; 95\% CI: $2.86,5.12 ; 6$ studies) and chronic $(1.45 \% ; 0.62,2.28 ; 2$ studies) intake and reduced systolic $(-5.88 \mathrm{~mm} \mathrm{Hg} ;-9.55$, $-2.21 ; 5$ studies $)$ and diastolic $(-3.30 \mathrm{~mm} \mathrm{Hg} ;-5.77,-0.83 ; 4$ studies) blood pressure. Soy protein isolate (but not other soy products or components) significantly reduced diastolic blood pressure $(-1.99 \mathrm{~mm} \mathrm{Hg} ;-2.86,-1.12 ; 9$ studies $)$ and LDL cholesterol $(-0.19 \mathrm{mmol} / \mathrm{L} ;-0.24,-0.14 ; 39$ studies $)$. Acute black tea consumption increased systolic $(5.69 \mathrm{~mm} \mathrm{Hg}$; $1.52,9.86 ; 4$ studies $)$ and diastolic $(2.56 \mathrm{~mm} \mathrm{Hg} ; 1.03$, $4.10 ; 4$ studies) blood pressure. Green tea reduced LDL $(-0.23 \mathrm{mmol} / \mathrm{L} ;-0.34,-0.12 ; 4$ studies $)$. For many of the other flavonoids, there was insufficient evidence to draw
\end{abstract}

Hooper L, Kroon PA, Rimm EB, et al.: Flavonoids, flavonoid-rich foods, and cardiovascular risk: a meta-analysis of randomized controlled trials. Am J Clin Nutr 2008, 88:38-50.

N. K. Hollenberg $(\bowtie)$

Departments of Medicine and Radiology,

Harvard Medical School, Brigham and Women's Hospital,

Mailstop PBB1-Rad Building, 15 Francis Street,

Boston, MA 02115, USA

e-mail: djpagecapo@rics.bwh.harvard.edu conclusions about efficacy. To date, the effects of flavonoids from soy and cocoa have been the main focus of attention. Future studies should focus on other commonly consumed subclasses (eg, anthocyanins and flavanones), examine doseresponse effects, and be of long enough duration to allow assessment of clinically relevant endpoints.

\section{Editor's Comments}

Many of us grew up in homes where mothers spent much time seeing to it that we ate our vegetables. Every set of guidelines on nutrition features the importance of eating fruit and vegetables. Why are fruit and vegetables healthy? Much less attention is paid to answering that fundamental question.

A large part of the answer may well lie in the area covered by this meta-analysis: flavonoid intake. There are thousands of flavonoids with a wide range of actions. One apparent feature that many of them share is activation of nitric oxide synthesis, which may well provide a large part of the benefit they produce.

Not mentioned in this review is a fundamental problem faced by those who use epidemiology — and specifically the history of ingesting various foods - as their source of information. For example, every cocoa pod is rich in flavonoids, but fermentation is required, and cocoa fermentation reduces the flavonoid content. As flavonoids are bitter, many of the best chocolates are treated with a maneuver to reduce their bitterness and thus their flavonoid content. A similar story applies to grapes and red wine. Grapes from the same vine sampled a year apart can show a striking difference in their flavonoid content and in the flavonoid content of the red wine produced from them. These factors can never be captured by history. What we need are assays of chemicals in blood and urine that will provide a reliable index of flavonoid content. 